IV.

Aus dem pharmakologisehen Institut zu Marburg.

\title{
Zur Kenntniss des Phlorhizindiabetes.
}

Von

Dr. Otto Loewi,

Privatdocent und Assistent des Institutes.

\section{Ueber den Einfluss der Applicationsweise des Phlorhizins anf die Grösse der Zuckerausscheidung.}

Bereits v. Mering ${ }^{1}$ ) hat festgestellt, dass nach subcutaner Injection von Phlorhizin mehr Zucker ausgeschieden wird als nach Eingabe per os. Es schien geboten, den Versuch unter gleichzeitiger Controle des $\mathrm{N}$-Umsatzes zu wiederholen, um zu prifen, ob nicht der in Folge subcutaner Injection etwa eintretende grössere Eiweissumsatz die Differenz erkläre.

Versuch 1 .

Weibl. Dogge, $17,67 \mathrm{~kg}$ schwer. Alle 24 Std. katheterisirt. Futter $500 \mathrm{~g}$ Fleisch. Wasser ad libitum.

\begin{tabular}{|c|c|c|c|c|c|c|c|c|}
\hline Datum & $\begin{array}{l}\text { Harn- } \\
\text { menge }\end{array}$ & $\begin{array}{l}\text { Spec. } \\
\text { Gew. }\end{array}$ & $\left.\mathrm{N}^{2}\right)$ & $\left.\mathrm{P}_{2} \mathrm{O}_{5}{ }^{3}\right)$ & Zucker ${ }^{4}$ ) & Bemerkungen & $\begin{array}{c}\text { Gew. } \\
\mathrm{kg}\end{array}$ & $\begin{array}{l}\text { Tem- } \\
\text { peratur }\end{array}$ \\
\hline $\begin{array}{r}1900.14 . \mathrm{I} \\
15 . \\
16 . \\
17 . \\
18 . \\
19 . \\
20 . \\
21 . \\
22 . \\
23 . \\
24 . \\
25 . \\
26 . \\
27 . \\
28 . \\
29 . \\
30 . \\
31 .\end{array}$ & $\begin{array}{l}290 \\
345 \\
480 \\
340 \\
420 \\
455 \\
480 \\
300 \\
320 \\
380 \\
500 \\
500 \\
470 \\
830 \\
490 \\
315 \\
400 \\
390\end{array}$ & $\begin{array}{l}1016 \\
1012 \\
1008 \\
1007 \\
1011 \\
1015 \\
1015 \\
1007 \\
1007 \\
1007 \\
1007 \\
1007 \\
1007 \\
1022 \\
1014 \\
1007 \\
1007 \\
1007\end{array}$ & $\begin{array}{l}12,94 \\
14,67 \\
14,17 \\
14,45 \\
14,50 \\
15,01 \\
15,12 \\
14,70 \\
15,68 \\
14,56 \\
15,08 \\
14,00 \\
15,83 \\
17,86 \\
16,97 \\
12,94 \\
15,57 \\
15,79\end{array}$ & $\begin{array}{l}1,74 \\
1,80 \\
1,66 \\
1,70 \\
1,64 \\
1,68 \\
1,76 \\
2,04 \\
1,84 \\
1,58 \\
1,44 \\
1,60 \\
1,30 \\
1,92 \\
2,00 \\
1,54 \\
1,80 \\
1,80\end{array}$ & $\begin{array}{r}18,72 \\
28,08 \\
6,00\end{array}$ & $\begin{array}{c}3 \times 2 \mathrm{~g} \text { Phlorhiz } \\
\text { subcutan }\end{array}$ & $\begin{array}{c}17,67 \\
17,80 \\
17,60 \\
17,70 \\
17,65 \\
17,60 \\
17,20 \\
17,27 \\
17,48 \\
17,57 \\
17,62 \\
17,67 \\
17,30 \\
16,90 \\
16,85 \\
17,05 \\
17,17 \\
17,25\end{array}$ & $\begin{array}{c}38,4 \\
38,6 \\
38,5 \\
38,9 \\
38,3 \\
38,4 \\
38,3 \\
38,5 \\
38,3 \\
38,3 \\
\mathbf{3 9 , 3} \\
\mathbf{3 9 , 5} \\
38,5 \\
38,3 \\
38,4\end{array}$ \\
\hline
\end{tabular}

1) Zeitschr. f. klin. Med. Bd. 16. S. 431. 1890.

2) $\mathrm{N}$ nach Kjeldahl.

3) Urantitration.

4) Polarisation vor und nach Vergährung. 
Nach Darreichung des Phlorhizines per os ist die Stickstoffausscheidung im Harn nicht wesentlich gestiegen, wohl aber nach subeutaner Injection. Doch lehrt die folgende Berechnung, dass die vermehrte Zuckerausscheidung im letzteren Fall nicht allein durch den grösseren Eiweissumsatz bedingt ist.

Die Zuckerausscheidung betrug nach Eingabe per os 52,8 g, nach Injection per eutem $126,4 \mathrm{~g}$. Die Stickstoffausfuhr stieg nach dem 1. Eingriff um höchstens $1 \mathrm{~g}$, nach dem 2. um ca. $5 \mathrm{~g}$.

Berechnen wir, dass aus 6,25 g Eiweiss entsprechend $1 \mathrm{~g}$ Harnstickstoff sich 6,5 g Zucker bilden können, d. i. die Menge, die sich ergiebt, wenn wir die unmögliche Annahme machen wollten, dass - abzüglich des Kohlenstoffs, der als Bestandtheil des Harnstoffs mit zur Ausseheidung gelangt - aller Kohlenstoff des Eiweisses in Zucker übergeht, so hätten wir nach subcutaner Injection $4 \times 6,5 \mathrm{~g}=26 \mathrm{~g}$ Zucker mehr zu erwarten, als nach der ersten Phlorhizingabe, also in Summa 78,8 g.

Thatsächlich wurden aber ausgeschieden 126,4 g; also sind $126,4-78,8=47,6 \mathrm{~g}$ mindestens nicht aus dem vermehrten Eiweisszerfall abzuleiten.

Auch der Glycogenvorrath des Thieres kann bei der zweiten Vergiftung nicht grösser angenommm werden als bei der ersten: die Ernährung war dieselbe geblieben wie bis dahin. Eher hätten wir Grund, in Folge der kurz vorher stattgehabten Glycogenentleerung bei der subcutanen Injection einen geringeren Stand desselben anzunehmen.

Wir müssen demnach für die grössere Intensität des Diabetes nach subcutaner Application des Giftes nach einem anderen Erklärungsmodus fahnden. Das nächstliegende wäre die Annahme einer unvollkommenen Resorption vom Darm aus. Diese Frage wurde bereits fruher von Moritz u. Praussnitz ${ }^{2}$ untersucht. Sie fanden indess nach einer Gabe von $1 \mathrm{~g}$ per os $\mathrm{k}$ ein unverändertes Phlorhizin im Koth. Ebenso berichtet $\mathrm{K} \mathfrak{u} l \mathrm{z}^{3}$ ), in verschiedenen Versuchen kein Phorhizin im Darm gefunden zu haben.

Da eine Spaltung des Phlorhizines im Darm stattfinden könnte und das wirksame in Wasser unlösliche Phloretiu sich bei der Suche

1) S. a. v. Mering, Zeitschr. f. kl. Med. Bd. 14. S. 405. 1888.

2) Zeitschr. f. Biol. Bd. 27. S. 81. 1890.

3) Külz u. Wright, Ebenda. Bd. 27. S. 181. 1890. 
nach Phlorhizin dem Nachweis entziehen könnte, wurde der folgende Versuch angestellt.

\section{Versuch 2.}

Foxterrier $12 \mathrm{~kg}$ schwer.

27. IV erhält $5 \mathrm{~g}$ Phlorhizin in Wasser aufgeschwemmt per Sonde.

29. IV. Fester Koth abgesetzt. Derselbe wird bei schwach essigsaurer Reaction unter Alkoholzusatz getrocknet, dann mit 96 Proc. Alkohol 3 mal heiss extrahirt. Das Extract wird mit Natriumcarbonat schwach alkalisirt und mit Wasser auf $100 \mathrm{ccm}$ aufgeftullt; davon werden einem $15 \mathrm{~kg}$ schweren, männlichen Hund, der noch nicht zu Versuchen benutzt war, $30 \mathrm{ccm}$ subcutan injicirt. Der in den nächsten 24 Stunden gelassene Harn, $295 \mathrm{ccm}$, enthălt 6 Proc. Zucker. Controlversuche mit Extract aus normalem Koth verliefen negativ:"

Das nicht injicirte Extract wird mit 5 proc. Schwefelsäure 2 Stunden am Rückflusskühler digerirt, das Filtrat wird neutralisirt, ergiebt minimale Reduction alkalischer Kupferlösung, ein Beweis, dass nur Spuren von unverändertem Phlorhizin im Koth waren. Der Filterrickstand bewirkt in $\mathrm{H}_{2} \mathrm{O}$ aufgeschwemmt und einem Hund injieirt starke Glycosurie.

Der Versuch beweist, dass im Darm die Resorption des Phlorhizines keine vollständige ist, dass ferner eine Spaltung stattfindet, wobei ein schwer resorbirbares, diabetisch machendes spaltungsproduct resultirt.

Um von andrer Seite zu zeigen, dass lediglich die Processe im Darm an der Minderausscheidung von Zucker nach Darreichung: des Phlorhizins per os schuld sind, wurde folgender Versuch unternommen.

\section{Versuch 3.}

20. V. 1901 . Weiblicher Dachshund $7,2 \mathrm{~kg}$ schwer. In leichter Aethernarkose wird eine Dünndarmschlinge hervorgeholt und in eine, peripher abgebundene kleine Mesenterialvene $0,006 \mathrm{~g}$ Phlorhizin in 1 ecm 1 proc. Natriumearbonatlösung injicirt. Die Schlinge wird reponirt. Glucose.

21. V. 1901. $600 \mathrm{cem}$ Harn entleert mit $1 ; 1$ Proc. $=6,6 \mathrm{~g}$ 
Das Ergebniss des Versuches lehrt, dass eine Phorhizinmenge, die nach anderweit gewonnenen Erfahrungen eben noch diabetisch macht, wirksam ist, wenn sie lediglich mit Umgehung des Darmes ins Blut gelangt. Die geringere Wirksamkeit des Giftes nach Eingabe per os wird also nicht durch Zerstörung oder Fixation in der Leber bedingt.

Nunmehr war festzustellen, ob zur Resorption gelangtes Phloretin sehwächer wirkt als $\mathrm{Phlorhizin.} \mathrm{Eine} \mathrm{diesbezïgliche}$ Angabe stammt bereits von v. Mering. Der folgende Versuch, der einen genaueren, quantitativen Vergleich zulässt, bestätigt sie.

$$
\mathrm{Versuch} 4 .
$$

Weiblicher Schäferhund $23,15 \mathrm{~kg}$ schwer.

Steht seit 20. V. unter constanter Phlorhizinwirkung. Alle 8 Stunden $1,5 \mathrm{~g}$ Phlorhizin und Fitterung mit $250 \mathrm{~g}$ Fleisch $+10 \mathrm{~g}$ Fett.

\begin{tabular}{|c|c|c|c|c|c|c|c|c|c|}
\hline \multirow{2}{*}{$\begin{array}{c}\text { Datum } \\
1901\end{array}$} & \multirow{2}{*}{ No. } & \multirow{2}{*}{$\mathrm{N}$} & \multicolumn{2}{|c|}{ Zucker } & \multirow{2}{*}{$D: \mathbf{N}$} & \multirow{2}{*}{$\begin{array}{l}\text { Harn- } \\
\text { menge }\end{array}$} & \multirow{2}{*}{$\begin{array}{c}\text { Wasser } \\
\text { getrunken }\end{array}$} & \multirow{2}{*}{ Gew. } & \multirow{2}{*}{ Bemerkungen } \\
\hline & & & Pol. ${ }^{1}$ ) & | Red. ${ }^{2}$ ) & & & & & \\
\hline \multirow[t]{3}{*}{$23 . \mathrm{V}$} & 1 & 9,74 & 25,74 & 30,9 & 2,7 & 50 & 420 & \multirow[t]{3}{*}{23,15} & \multirow{5}{*}{$0,9 \mathrm{~g}$ Phloretin $\left.{ }^{3}\right)$} \\
\hline & 2 & 9,18 & 29,64 & 34,6 & 3,2 & 200 & 360 & & \\
\hline & 3 & 8,68 & 28,86 & 30,2 & 3,3 & 175 & 330 & & \\
\hline \multirow[t]{2}{*}{ 24. V } & 1 & 9,60 & 16,40 & 18,0 & 1,8 & 150 & 280 & \multirow[t]{2}{*}{23,1} & \\
\hline & 2 & 8,2 & 30,40 & 30,9 & 3,7 & 170 & 370 & & \\
\hline
\end{tabular}

\section{Ueber den Einfluss der Dosirung auf die Grösse der Zuckerausscheidung.}

Die eben noch diabetisch machende Einzelgabe von subcutan injicirtem Phlorhizin liegt bei Hunden von $7-23 \mathrm{~kg}$ Gewicht zwischen 4 u. $6 \mathrm{mg}$. Steigt man, so erzielt man bei einer bestimmten Gabe eine Zuckerausscheidung, die sich unter sonst gleichen Bedingungen durch die Injection grösserer Phlorhizinmengen nicht weiter erhöhen lässt, wie das die folgenden Versuche zeigen.

1) Vor und nach Vergährung.

2) Nach Allihn.

3) Entsprechend 1,5 g Phlorhizin und statt dessen subcutan injicirt. Das Präparat batte ich nach den Angaben von Stas dargestellt. Es zeigte dieselben Eigenschaften wie das von Moritz und Praussnitz (l. c.) nach derselben Vorschrift hergestellte. 


$$
\text { Versuch } 5 \text {. }
$$

Weiblicher Schäferhund $22,65 \mathrm{~kg}$ schwer.

Seit 4. VI. 1901 Hunger.

Seit 5. VI. 1901 alle 8 Stunden $1,5 \mathrm{~g}$ Phlorhizin subcutan und $\mathrm{Ab}$ nabme des Harns mittelst Katheters.

\begin{tabular}{|c|c|c|c|c|c|c|c|c|c|c|c|}
\hline \multirow[b]{2}{*}{$\begin{array}{c}\text { Datum } \\
1901\end{array}$} & \multirow[b]{2}{*}{ No. } & \multicolumn{2}{|l|}{$\mathbf{N}$} & \multicolumn{2}{|c|}{ Zucker } & \multirow[b]{2}{*}{$\mathrm{D}: \mathbf{N}$} & \multirow{2}{*}{$\begin{array}{l}\text { Wasser- } \\
\text { auf- } \\
\text { nahme }\end{array}$} & \multirow[b]{2}{*}{$\begin{array}{l}\text { Harn- } \\
\text { menge }\end{array}$} & \multirow[b]{2}{*}{$\begin{array}{l}\text { Phlor- } \\
\text { hizin }\end{array}$} & \multirow[b]{2}{*}{ Nahrg. } & \multirow[b]{2}{*}{ Gew. } \\
\hline & & $\begin{array}{c}\text { pro } \\
\text { Periode }\end{array}$ & $\begin{array}{c}\text { pro } \\
\text { die }\end{array}$ & $\begin{array}{l}\text { pro } \\
\text { Per. }\end{array}$ & $\begin{array}{l}\text { pro } \\
\text { die }\end{array}$ & & & & & & \\
\hline 9. VI & $\begin{array}{l}1 \\
2 \\
3\end{array}$ & $\begin{array}{l}2,55 \\
2,77 \\
2,98\end{array}$ & 8,30 & $\begin{array}{l}10,53 \\
11,16 \\
10,92\end{array}$ & 32,61 & $\begin{array}{l}4,1 \\
4,0 \\
3,9\end{array}$ & $\begin{array}{r}25 \\
125 \\
75\end{array}$ & $\begin{array}{l}165 \\
175 \\
170\end{array}$ & $\begin{array}{l}1,5 \\
= \\
=\end{array}$ & $\begin{array}{c}\text { Hunger } \\
= \\
=\end{array}$ & 22,65 \\
\hline 10. VI & $\begin{array}{l}1 \\
2 \\
3\end{array}$ & $\begin{array}{l}3,15 \\
3,29 \\
3,22\end{array}$ & 9,66 & \begin{tabular}{|c|}
10,92 \\
10,53 \\
10,92
\end{tabular} & 32,37 & $\begin{array}{l}3,4 \\
3,2 \\
3,3\end{array}$ & $\begin{array}{l}95 \\
75 \\
85\end{array}$ & $\begin{array}{l}170 \\
185 \\
190\end{array}$ & $\begin{array}{l}= \\
2,5 \\
2,5\end{array}$ & $\begin{array}{l}= \\
= \\
=\end{array}$ & 22,55 \\
\hline
\end{tabular}

Versuch 6.

Derselbe Hund. Seit Ablauf des vorigen Versuches andauernd unter Phlorhizinwirkung stehend. Anordnung wie oben.

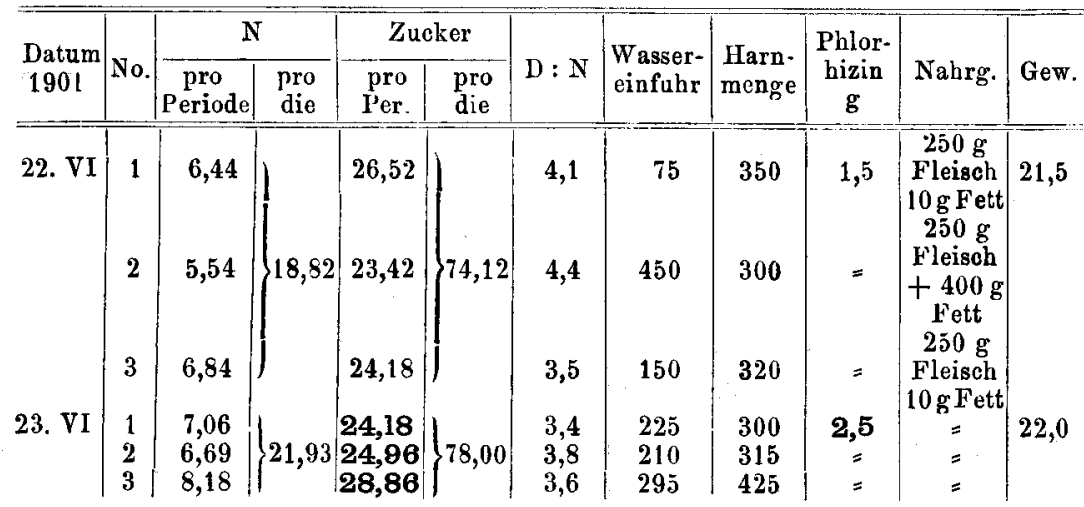

Versuch 7.

Derselbe Hund. Noch immer unter Phlorhizinwirkung. Anordnung wie oben.

\begin{tabular}{c|c|c|c|c|c|c|c|c|c}
\hline $\begin{array}{c}\text { Datum } \\
1901\end{array}$ & No. & N & Zucker & D : N & $\begin{array}{c}\text { Wasser- } \\
\text { einfuhr }\end{array}$ & $\begin{array}{c}\text { Harn- } \\
\text { menge }\end{array}$ & $\begin{array}{c}\text { Phlor- } \\
\text { hizin } \\
\mathrm{g}\end{array}$ & Nahrung & Gew. \\
\hline & & & &
\end{tabular}


Die Erhöhung der Phlorhizindose von 1,5 g auf 2,5 $\mathrm{g}$ und auf $5 \mathrm{~g}$ hat beim hungernden, wie bei dem mit $250 \mathrm{~g}$ Fleiseh und $10 \mathrm{~g}$ Fett ernährten Thier die Zuckerausscheidung nicht gesteigert. Darnach sollte man annehmen, dass $1,5 \mathrm{~g}$ Phlorhizin die maximal vergiftende Dose für dieses Thier ist. Dass dem aber nicht so ist, beweist der Vergleich der beiden folgenden Versuche.

$$
\mathrm{Versuch} 8 .
$$

Dasselbe Thier. Anordnung wie oben.

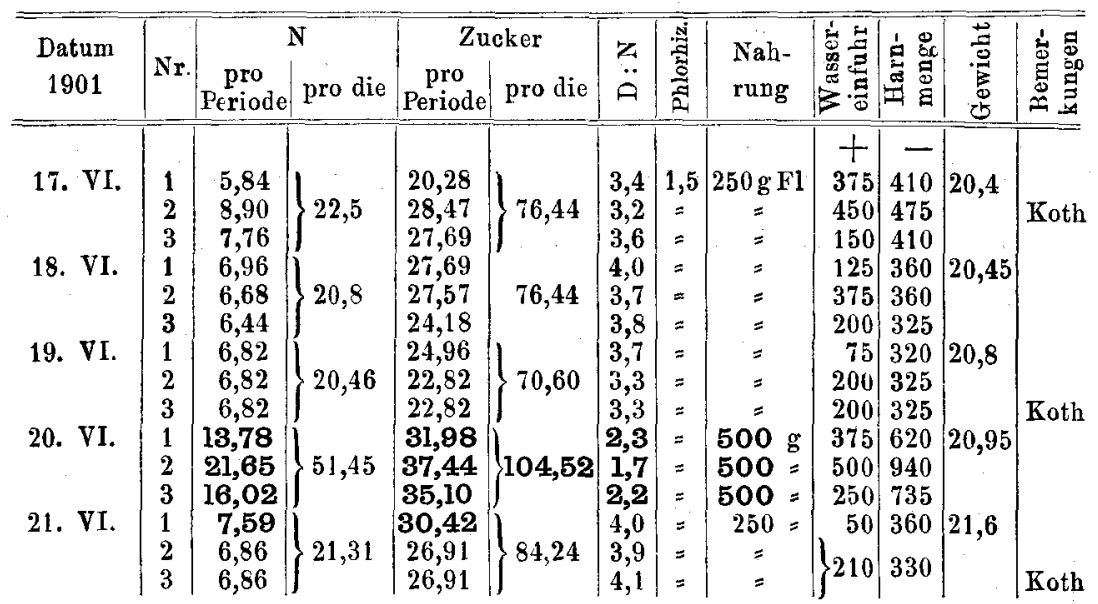

Besprechung des Versuches.

Als, wie aus der Tabelle des Versuches ersichtlich ist, am 20. VI die einzelne Fleischration verdoppelt wurde, stieg die Zuckerausscheidung zwar an, aber durchaus nicht entsprechend der Erhöhung der N-Ausfuhr als Maass des umgesetzten Eiweisses, wie sich das aus der Betrachtung des Verhältnisses D:N ergiebt.

War es auch nach dem Ausfall der Versuche 5-7 a priori unwahrscheinlich, dass durch eine Steigerung der Phlorhizindose sich mehr Zucker in den Harn treiben liesse, so machte ich doch den folgenden Controlversuch. 


$$
\text { Versuch } 9 .
$$

Dasselbe Thier. Anordnung wie oben.

\begin{tabular}{|c|c|c|c|c|c|c|c|c|c|c|c|c|}
\hline Datum & & 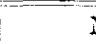 & N & Zur & ker & $z$ & 苞 & Nab- & $\dot{\Delta}$ & $\therefore \infty$ & 경 & 눙뭉 \\
\hline 1901 & $\mathrm{Nr}$. & $\left|\begin{array}{c}\text { pro } \\
\text { Periode }\end{array}\right|$ & pro die & $\begin{array}{c}\text { pro } \\
\text { Periode }\end{array}$ & pro die & $\ddot{\theta}$ & 泀 & rung & 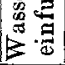 & 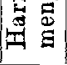 & $\underbrace{0}_{0}$ & 苟 \\
\hline 23. VI. $\left.{ }^{3}\right)$ & $\begin{array}{l}1 \\
2 \\
3\end{array}$ & $\begin{array}{l}7,06 \\
6,69 \\
8,18\end{array}$ & 21,93 & $\begin{array}{l}24,18 \\
24,96 \\
28,86\end{array}$ & 78,0 & $\begin{array}{l}3,4 \\
3,8 \\
3,6\end{array}$ & $\begin{array}{c}2,5 \\
= \\
=\end{array}$ & $\begin{array}{c}250 \mathrm{~g} F \mathbf{F l} \\
= \\
=\end{array}$ & $\begin{array}{l}225 \\
210 \\
295\end{array}$ & $\begin{array}{l}300 \\
315 \\
425\end{array}$ & 22,0 & \\
\hline 24. VI. & $\begin{array}{l}1 \\
2 \\
3\end{array}$ & $\begin{array}{l}14,95 \\
12,23 \\
16,25\end{array}$ & 53,43 & $\begin{array}{l}42,12 \\
51,48 \\
43,29\end{array}$ & 136,89 & $\begin{array}{l}2,9 \\
2,3 \\
2,6\end{array}$ & $\begin{array}{c}5 \\
5 \\
2,5\end{array}$ & $\begin{array}{l}500 \mathrm{~g} \\
500= \\
500=\end{array}$ & $\begin{array}{l}725 \\
500 \\
250\end{array}$ & $\begin{array}{r}750 \\
1100 \\
880\end{array}$ & 22,2 & Koth \\
\hline 25. VI. $\left.{ }^{1}\right)$ & $\begin{array}{l}1 \\
2 \\
3\end{array}$ & $\begin{array}{l}7,78 \\
8,90 \\
8,31\end{array}$ & 24,99 & $\begin{array}{l}29,64 \\
31,20 \\
30,42\end{array}$ & 91,26 & $\begin{array}{l}3,8 \\
3,5 \\
3,7\end{array}$ & $\begin{array}{c}2,5 \\
= \\
=\end{array}$ & $\begin{array}{c}250 \mathrm{gFl} . \\
=\end{array}$ & $\begin{array}{l}270 \\
250 \\
250\end{array}$ & $\begin{array}{l}450 \\
450 \\
430\end{array}$ & 22,9 & \\
\hline
\end{tabular}

In diesem Versuch wurde bei Ernährung mit $500 \mathrm{~g}$ Fleisch und gleichzeitiger Steigerung der Phlorhizindose $30 \mathrm{~g}$ Zucker mehr ausgeschieden als im vorigen Versuch (8) bei derselben Nahrung und jener Phlorhizingabe, die bei $250 \mathrm{~g}$ Fleiseh maximal wirksam war. Dabei war die $\mathrm{N}$-Ausscheidung beidemale annähernd gleich (51,4 gegen 53,4), sodass dass $\mathrm{D}: \mathrm{N}$, nicht unter 2,3 (gegen 1,7 in Versuch 8) herabging und es scheint fraglich, ob nicht bei weiterer Steigerung der Dose noch mehr Zucker erschienen und das Verhältniss 3,8 erreicht worden wäre.

Aus dem Ausfall des Versuches ergiebt sich der für Theorie und Praxis der Phlorhizinwirkung wichtige Schluss, dass ein totaler Diabetes nur dann eintritt, wenn mit Erhöhung der zur Zuckerbildung verwandten Zufuhr, auch die Phlorhizindose gesteigert wird. Mit andern Worten: Mit einer bestimmten Dose vergiftet man nur für eine gewisse Menge zuckerliefernder Nahrung, nicht total.

\section{Ueber den Einfluss der Körperwärme auf die Grösse der Zuckerausscheidung.}

Da sich Temperaturerhöhungen bei einer andauernden Injectionsreihe kaum vermeiden lassen, schien es mir zweckmässig, einen Versuch unter genauer Controle dieses Factors auszuführen.

1) Die Werthe dieses Tages sind übersichtshalber noch einmal mitgetheilt. 
Versuch 10.

Weibl. Dogge. $500 \mathrm{~g}$ Fleisch, $250 \mathrm{cem} \mathrm{H}_{2} \mathrm{O}$.

\begin{tabular}{|c|c|c|c|c|c|c|c|c|c|c|}
\hline No. & $\begin{array}{c}\text { Datum } \\
1900\end{array}$ & Menge & s. G. & N. & $\mathrm{P}_{2} \mathrm{O}_{5}$ & $\begin{array}{r}\text { Glv } \\
\text { Pol. }\end{array}$ & $\begin{array}{l}\text { cose } \\
\text { Allihn }\end{array}$ & 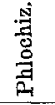 & Temp. & $D: N$ \\
\hline 1 & 13. I & & & & & & & & & \\
\hline 2 & 14. & 450 & 1017 & 14,4 & 1,9 & & & & 38,3 & \\
\hline 3 & 15. & 370 & 1017 & 15,7 & 1,9 & & & & 38.6 & \\
\hline 4 & 16. & 355 & 1017 & 15,8 & 1,96 & & & & 38,5 & \\
\hline 5 & 17. & 340 & 1017 & 15,5 & 1,94 & & & & 38,4 & \\
\hline 6 & 18. & 330 & 1017 & 15,3 & 1,94 & & & & 38,4 & \\
\hline 7 & 19. & 700 & 1044 & 16,9 & 1,88 & 67,1 & 68,3 & 4,5 & 40,2 & 4,0 \\
\hline 8 & 20. & 730 & 1044 & 20,2 & 2,0 & 78,2 & 80,1 & $=$ & 39,3 & 3,9 \\
\hline 9 & 21. & 780 & 1057 & 23,8 & 2,47 & 75.0 & 76,5 & $=$ & 38,7 & 3,1 \\
\hline 10 & 22. & 690 & 1054 & 22,4 & 2,5 & 76,2 & 80,2 & $\approx$ & 39,0 & $\mathbf{3 , 5}$ \\
\hline 11 & 23. & 700 & 1051 & 21,3 & 2,3 & 74,1 & 76,3 & $=$ & 39,0 & 3,5 \\
\hline 12 & 24. & 600 & 1045 & 19,9 & 2,3 & 60,0 & 64,0 & $=\cdot$ & 39,0 & 3,1 \\
\hline 13 & 25. & 610 & 1044 & 19,0 & 2,3 & 58,1 & 63,0 & $=$ & 38,5 & 3,1 \\
\hline 14 & 26. & 600 & 1040 & 18,7 & 2,2 & 61,3 & 63,3 & $=$ & 39,3 & 3,3 \\
\hline 15 & 27. & 625 & 1042 & 18,2 & 1,8 & 76,0 & 78,1 & 9,0 & 39,6 & 4.0 \\
\hline 16 & 28. & 690 & 1054 & 22,5 & 2,5 & 77,2 & & 9,0 & 40,2 & 3,4 \\
\hline 17 & 29. & 620 & 1048 & 21,0 & 2,2 & 72,2 & & & 39,0 & 3,4 \\
\hline 18 & 30. & 520 & 1030 & 21,6 & & 18,0 & & & & \\
\hline 19 & 31. & 350 & 1020 & & & & & & & \\
\hline
\end{tabular}

Eine gesetzmässige Abhängigkeit der Grösse der Zuckerausscheidung von den Temperaturschwankungen ist nicht erkennbar. ${ }^{1}$ )

\section{Ergebnisse.}

1. Der Untersohied in der Intensität der Phlorhizinglycosurie nach innerer und subcutaner Anwendung des Giftes ist durch Nichtresorption eines Theiles des Giftes bez. dessen wirksamer Spaltungsproducte im Darmcanal bedingt.

2. Die Phlorhizindose, durch deren Steigerung bei bestimmter Ernährung eine Erhöhung der Zuckerausfuhr nicht mehr bewirkt wird, vergiftet nicht maximal; vielmehr ist hierzu bei steigender Zufuhr von zuckerbildendem Material eine Erhöhung der Phlorhizindose nöthig.

3. Aenderung der Eigentemperatur ist ohne wesentliohen Einfluss auf die Grösse der Phlorhizinglycosurie.

1) Nebelthau (Arch. f. exper. Pathol. u. Phara. Bd. 46. S. 385. 1901) findet auch keinen merklichen Einfluss des Fiebers auf die Zuckerausscheidung nach Pankreasexstirpation. 